\title{
Anti-Hyperlipidemic Effect of Flavone-Rich Belamcanda chinensis (L.) DC. (Iridaceae) Leaf Extract in ICR Mice Fed High-Fat Diet
}

\author{
Hai-Wei Zhao ${ }^{1}$, Fang Lv ${ }^{1,2}$, Wei-Wei Meng ${ }^{1,2}$, Hao Dang ${ }^{1}$, Zhi-Long Sun ${ }^{1}$, Yan \\ Chen $^{1,2}$, Rong-Ji Dai ${ }^{1,2}$, Yu-Lin Deng ${ }^{1,2}$ and Chong-Ming Wu ${ }^{3 *}$ \\ ${ }^{1}$ School of Life Science, Beijing Institute of Technology, Beijing 100081, ${ }^{2}$ Beijing BIT\&GY Pharmaceutical R\&D, Beijing 100081, \\ ${ }^{3}$ Institute of Medicinal Plant Development, Chinese Academy of Medical Science \& Peking Union Medical College, Beijing \\ 100193, PR China
}

*For correspondence: Email: wucm1979@gmail.com; Tel: +86 10 68949331; Fax: +86 1068467208

\begin{abstract}
Purpose: To assess the anti-hyperlidemic effect of flavone-rich B. chinensis leaf extract (HTP) in ICR mice fed a high-fat diet.

Methods: HTP (100, 200 and $400 \mathrm{mg} / \mathrm{kg})$ were orally administered to ICR mice fed high-fat diet for 7 weeks. Blood glucose, as well as serum and hepatic levels of lipids were determined at the end of the experiment. Phosphorylation of adenosine 5'-monophosphate (AMP)-activated protein kinase (AMPK) and protein level of peroxisome proliferator-activated receptor a (PPAR $\alpha$ ) were analyzed by Western blot and enzyme-linked immunosorbent assay (ELISA) kit, respectively.

Results: Treatment with HTP significantly decreased peri-epididymal fat weight $(p<0.01$ and $p<0.05$ for 200 and $100 \mathrm{mg} / \mathrm{kg}$, respectively), lowered serum and hepatic lipid, and decreased glucose area under curve (AUC) in oral glucose tolerance test $(p<0.01 \mathrm{for} 200 \mathrm{mg} / \mathrm{kg})$. Western blot and ELISA analysis showed that administration of HTP $(200 \mathrm{mg} / \mathrm{kg})$ significantly increased AMPK $(p<0.05)$ phosphorylation and PPARa expression in liver $(p<0.05)$.

Conclusion: HTP can alleviate hyperlipidemia, at least in part, by up-regulation of AMPK and PPARa.
\end{abstract}

Keywords: Belamcanda chinensis, Flavone, Hyperlipidemia, Adenosine 5'-monophosphate-activated protein kinase, Peroxisome proliferator activated receptor-alpha, Glucose tolerance

Tropical Journal of Pharmaceutical Research is indexed by Science Citation Index (SciSearch), Scopus, International Pharmaceutical Abstract, Chemical Abstracts, Embase, Index Copernicus, EBSCO, African Index Medicus, JournalSeek, Journal Citation Reports/Science Edition, Directory of Open Access Journals (DOAJ), African Journal Online, Bioline International, Open-J-Gate and Pharmacy Abstracts

\section{INTRODUCTION}

The incidence of hyperlipidemia is currently increasing at a dramatic rate throughout the world [1]. The close relationship between elevated circulating levels of free fatty acids (FFA), triglycerides (TG) and LDL-cholesterol (LDL-C) and cardiovascular diseases (CVD) has been well documented [2,3] Although current pharmaceutics such as statins can effectively alleviate hyperlipidemia, they are often associated with undesirable side effects such as gastrointestinal discomfort, hyperglycemia and myopathy [4,5]. Dietary intervention through intake of polyphenol-rich plant products that can modulate lipid metabolism represents another modality of management $[6,7]$.

AMP-activated protein kinase (AMPK) and peroxisome proliferator-activited receptor $\alpha$ (PPARa) are key regulators of lipid metabolism. AMPK is activated by phosphorylation at 
threonine (Thr172) on the alpha-subunit This in turn promotes ATP-generating mechanisms such as lipid oxidation while decreasing energyconsuming processes like TG and protein synthesis $[8,9]$. On the other hand, activation of PPAR a by ligands up-regulates the expression of genes involved in fatty acid transport and oxidation [10,11]. AMPK and PPARa were proposed as major therapeutic targets for hyperlipidemia $[12,13]$.

Belamcanda chinensis belongs to the family of Iridaceae and their rhizomes have been widely used as traditional medicine in China. Many isoflavones such as tectoridin, iridin, irigenin and tectorigenin have been identified from this plant [14]. The antifebrile, antioxidant, antiinflammatory and hepato-protective activities of $B$. chinensis have been well-documented [15-17]. Previously, we reported the hypoglycemic and anti-hyperglycemic effects of flavone-rich $B$. chinensis leaf extract (HTP) in normal and STZinduced diabetic rats $[18,19]$. Apart from these, no study to-date has investigated the antihyperlipidemia activity of HTP. In this study, we assessed the anti-hyperlipidemia effects of HTP on high-fat diet induced obese mice. The stimulating effects of HTP on AMPK and PPARa were also studied.

\section{EXPERIMENTAL}

\section{Plant materials}

Leaves of Belamcanda chinensis were collected from Hainan province, South China, and authenticated by $\mathrm{Dr}$ Hubiao Chen (Health Science Center, Peking University, China). The preparation of HTP was performed as previously reported $[18,19]$. A voucher specimen has been deposited in School of Life Science, Beijing Institute of Technology (NO. HTP20120911).

\section{Animals and experiment design}

Male ICR mice $(20 \pm 2 \mathrm{~g}$, Peking University Laboratory Animal Center, Beijing, China) were housed at $22 \pm 2{ }^{\circ} \mathrm{C}$ and $55 \pm 5 \%$ relative humidity; $12 \mathrm{~h}$ light-dark cycle and allowed free access to water and feed. The study was carried out in accordance with International Guidelines for Care and Use of Laboratory Animals [20] and approved by Animal Ethical Committee of Beijing Institute of Technology (reg. no. 201209007/BITAEC).

Seventy-two ICR mice were kept in a humiditycontrolled room on a 12-h light-dark cycle with food and water available ad libitum for one week. Animals were then randomly divided into 6 groups with 12 mice in each group: BC, fed a standard diet and received $0.5 \%$ sodium carboxymethylcellulose (CMC-Na) solution only; $\mathrm{NC}$, fed a high-fat diet (HFD) and received $0.5 \%$ CMC-Na solution only; PC, fed a HFD and treated with gemfibrozil $(200 \mathrm{mg} / \mathrm{kg})$; and HTP groups, fed a HFD and treated with HTP (100, 200 and $400 \mathrm{mg} / \mathrm{kg}$, respectively). HFD consists of $10 \%$ lard, $10 \%$ sugar, $10 \%$ egg York, $1 \%$ cholesterol, $0.2 \%$ sodium cholate, and $68.8 \%$ standard diet. HTP and gemfibrozil were administrated by oral gavage. Body weight was monitored weekly. During the experiment, blood was collected from tail vein for the measurement of blood glucose. At the end of the 7th week, blood sample was collected from orbital venous plexus and the serum was prepared for biochemical analysis. Liver and peri-epididymal fat were removed, weighted and stored at $-70{ }^{\circ} \mathrm{C}$ refrigerator (Thermo Fisher Scientific, Waltham, USA,). Serum and hepatic levels of triglyceride (TG) and total cholesterol (TCH) were determined by corresponding kits (Nanjing Jiancheng Bioengineering Institute, Nanjing, China) according to the manufacturer's instruction. The hepatic PPARa level was determined by a mouse PPAR- $\alpha$ ELISA kit (HanKe Biotech co., Ltd, Beijing, China) according to the manufacture's instruction.

\section{Oral glucose tolerance test (OGTT)}

Animals were orally administrated corresponding regents after overnight fast. PC group was treated with acarbose $(50 \mathrm{mg} / \mathrm{kg})$. After another 2 $\mathrm{h}$, glucose $(2.5 \mathrm{~g} / \mathrm{kg})$ was given to each animal orally. Blood were collected from tail vein of each mouse at $0,30,60$, and 120 min after glucose administration, and glucose levels were determined by a blood glucose meter (Roche Diagnostics, Basel, Switzerland).

\section{Western blot}

Western blot analysis was performed on liver tissue extract as previously reported [21]. Antibodies against phospho-AMPKa (Thr172) and AMPKa were from Cell Signaling Technology Inc. (Beverly, USA), and antibody against GAPDH was from Abcam, Inc. (Cambridge, USA).

\section{Statistical analysis}

Data are presented as the means \pm S.D. Oneway ANOVA was used to determine significant 
differences among groups, after which the modified Student's t-test with the Bonferroni correction was used for comparison between individual groups. $P<0.05$ was considered statistically significant.

\section{RESULTS}

\section{Effect of HTP on peri-epididymal fat weight}

Animals in all groups showed a steady increase in body weight throughout the experimental period. At the end of the study, the average body weight of $\mathrm{NC}$ group was significantly higher than BC group $(p<0.05)$. Treating with HTP for 7 weeks did not influence the weight gain but significantly decreased the peri-epididymal fat weight and fat index (Table 1).

\section{Effect of HTP on blood and liver lipid profiles}

In animals fed a HFD (NC group), the serum levels of triglycerides (TG), total cholesterol (TCH), HDL-C, LDL-C and hepatic levels of TG, $\mathrm{TCH}$ were all significantly increased as compared with those fed a standard diet (BC group) (Fig. 1). Treatment with HTP (100, 200, $400 \mathrm{mg} / \mathrm{kg}$ ) or gemfibrozil (200 $\mathrm{mg} / \mathrm{kg}$ ) for 7 weeks significantly decreased the serum and hepatic levels of TG (Fig. 1). HTP did not lower serum $\mathrm{TCH}$ but significantly decreased hepatic $\mathrm{TCH}$ level at 200 and $400 \mathrm{mg} / \mathrm{kg}$. After 7 weeks of HTP or gemfibrozil treatment, serum HDL-C was increased and LDL-C decreased, but only gemfibrozil-treated group showed significant increase in serum HDL-C level when compared with the vehicle control.

Table 1: Effect of HTP on the body weight, peri-epididymal fat weight and fat index

\begin{tabular}{llll}
\hline Group (n=12) & $\begin{array}{l}\text { Body weight } \\
\mathbf{( g )}\end{array}$ & $\begin{array}{l}\text { Peri-epididymal fat } \\
\text { weight } \mathbf{( g )}\end{array}$ & $\begin{array}{l}\text { Fat index } \\
\text { (mg/g BW) }\end{array}$ \\
\hline BC & $44.8 \pm 4.3$ & $0.40 \pm 0.16^{* *}$ & $8.14 \pm 2.62^{* *}$ \\
NC & $46.1 \pm 3.0$ & $0.83 \pm 0.30 \# \#$ & $18.07 \pm 6.74 \# \#$ \\
PC & $43.1 \pm 2.3^{*}$ & $0.55 \pm 0.18^{*}$ & $12.70 \pm 3.84$ \\
HTP 100 & $44.8 \pm 3.9$ & $0.59 \pm 0.42$ & $9.57 \pm 3.15^{\star *}$ \\
HTP 200 & $44.8 \pm 3.6$ & $0.39 \pm 0.25^{\star *}$ & $8.62 \pm 5.33^{* *}$ \\
HTP 400 & $45.4 \pm 2.8$ & $0.57 \pm 0.29^{*}$ & $11.13 \pm 5.06^{*}$ \\
\hline
\end{tabular}

$B C=$ fed a standard diet; NC = fed a high fat-diet (HFD); PC = fed a HFD and treated with gemfibrozil (200 $\mathrm{mg} / \mathrm{kg}) ;$ HTPs = fed a HFD and treated with indicated dose of HTP. Values are mean \pm S.D; ${ }^{\#} p<0.01$ NC vs. $B C ;{ }^{*} p<0.05,{ }^{* *} p<0.01$ vs. NC

A

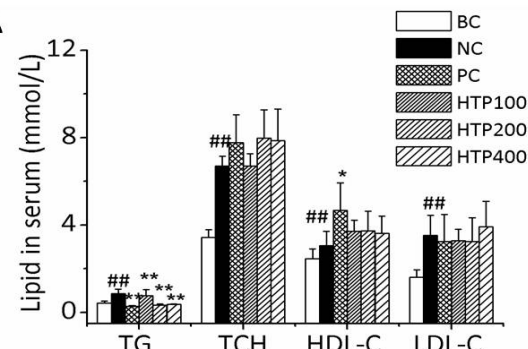

C

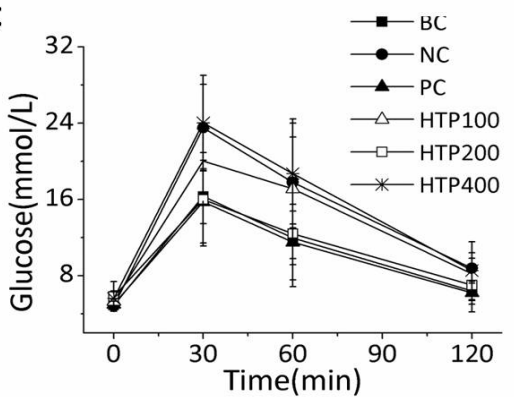

B

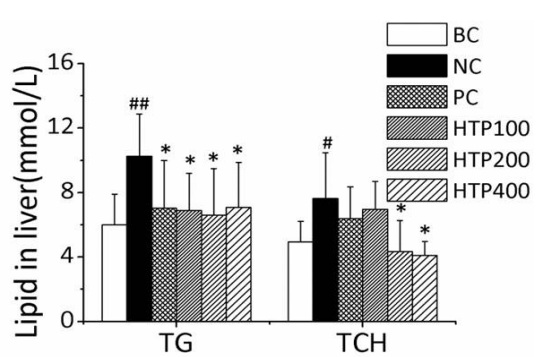

D

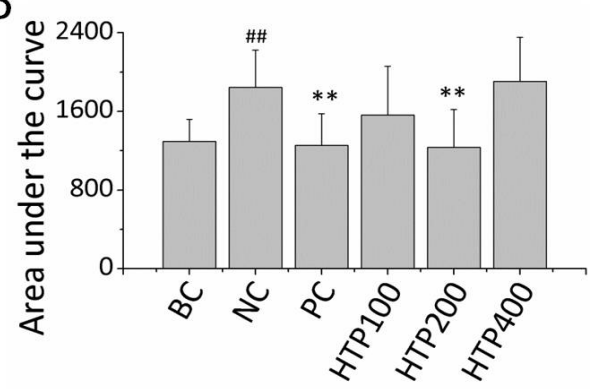

Figure 1: Effects of HTP on serum (A) and hepatic (B) lipid profiles and glucose tolerance $(C$ and $D)$ at the end of $7^{\text {th }}$ week. BC, fed a standard diet; NC, fed a high fat-diet (HFD); PC, fed a HFD and treated with gemfibrozil (200 $\mathrm{mg} / \mathrm{kg}$ ) or acarbose $(50 \mathrm{mg} / \mathrm{kg}$ for OGTT); HTPs, fed a HFD and treated with indicated dose of HTP. Values are mean \pm S.D. ${ }^{\#} p<0.05,{ }^{\#} p<0.01$ NC vs. BC; ${ }^{*} p<0.05,{ }^{* *} p<0.01$ vs. NC 
A
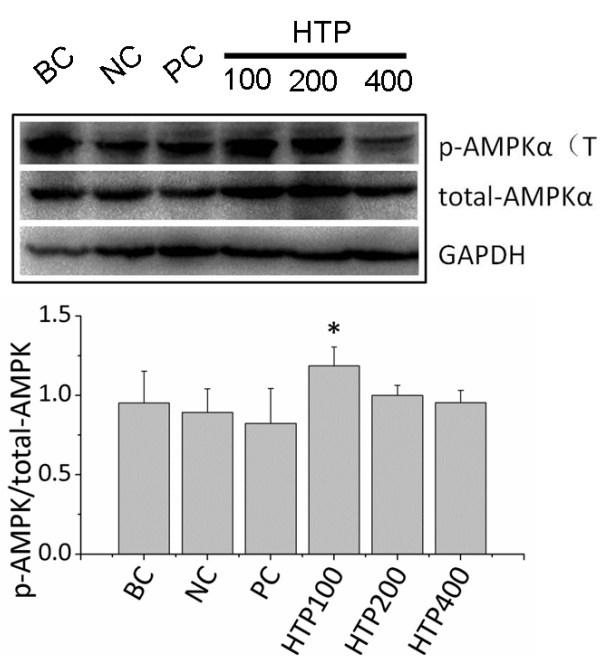

B

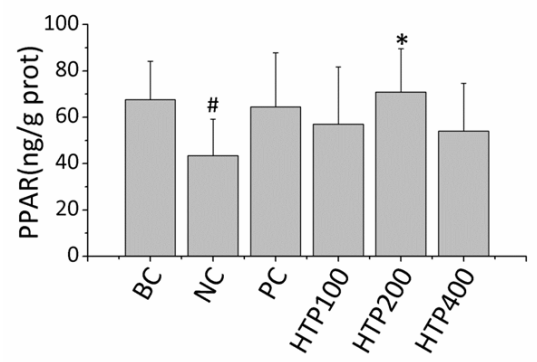

Figure 2: Effect of HTP on AMPK phosphorylation (A) and PPARa expression (B) in the liver of ICR mice. $B C$, fed a standard diet; NC, fed a high fat-diet (HFD); PC, fed a HFD and treated with gemfibrozil (200 $\mathrm{mg} / \mathrm{kg}$ ); HTPs, fed a HFD and treated with indicated dose of HTP. Values present as mean \pm S.D; ${ }^{*} p<0.05$ $\mathrm{NC}$ vs. $\mathrm{BC} ;{ }^{*} p<0.05$ vs. NC

\section{Effect of HTP on AUC in OGTT}

In oral glucose test (OGTT), acarbose-treated group (PC) and HTP200 group showed significant reduction in blood glucose levels at 30, 60 and 120 min (HTP200: $p<0.01$; PC: $p<$ $0.001)$. The area under the curve (AUC) of $P C$ group and HTP200 group was significantly lower than that of NC group $(p<0.01)$ (Fig.1).

\section{Effect of HTP on AMPK phosphorylation and PPAR $\alpha$ expression}

To ascertain the potential mechanism of HTPmediated anti-hyperlipidemic effect, the levels of AMPKa and its phosphorylated form ( $p$-AMPKa Thr172) in liver were measured by western-blot analysis. As shown in Fig. 2, the expression level of p-AMPKa (Thr172) in HFD-fed mice was lower than that in standard diet-fed animals. Treatment with HTP up-regulate the expression of pAMPKa (Thr172) $(p<0.05)$. ELISA analysis showed that HTP also increased the expression of PPARa in the liver (Fig. 2).

\section{DISCUSSION}

Belamcanda chinensis is a traditional Chinese medicine used for the treatment of cough and pharyngitis. In Hainan Province, South China, the local residents take the decoction of Belamcanda chinensis leaves as a folk medicine to cure diabetes-related hyperlipidemia. Our previous study has demonstrated the hypoglycemic activity of total flavones from Belamcanda chinensis leaves (HTP) $(18,19)$. Five compounds have been identified in HTP which are 2"-Orhamnosyl swertisin, swertisin, tectoridin, iristectoriginin $A$, and iridin $[19,22]$. Previous studies [23,24] have demonstrated the hypolipidemic and anti-dyslipidemic activity of flavonoid-rich extract, revealing a potential role of flavonoids in the treatment of hyperlipidemia. Lee et al [25] and Xiong et al [26] have reported the hypolipidemic effects of tectorigenin and tectoridin isolated from Pueraria thunbergiana and Pueraria lobata, respectively. These two flavonoids also exist in $B$. chinensis leaves [19]. Apart from these, no direct evidence for the antihyperlipidemic activity of $B$. chinensis leaf extract has been published up to now. In this work, we demonstrated for the first time that the $B$. chinensis leaf extract (HTP) possessed potent antihyperlipidemic activities in ICR mice fed a HFD.

Our results presented in this work showed that HTP can alleviate hyperlipidemia from several aspects. Firstly, treatment with HTP did not influence the body weight gain but significantly decreased peri-epididymal fat weight. This beneficial effect of HTP was not due to the decrease of food intake as HTP-treated mice showed a similar food intake in our experiment (data not shown). Secondly, treatment with HTP significantly improved the lipid profiles in serum and liver. It preferred decreasing the levels of triglyceride in both serum and liver. Finally, HTP also remarkably decreased the postprandial hyperglycemia, reducing the glucose AUC after OGTT, which suggested the beneficial effect of HTP on insulin sensitivity.

AMPK and PPARa have been broadly reported to be potential therapeutic target of fenofibrate for the treatment of dyslipidemia [27]. In order to clarify the potential mechanism under HTPmediated antihyperlipidemc effect, the influence of HTP on the expression of these two key lipid 
metabolic regulators was investigated. Our data showed that administration of HTP significantly increased the expression levels of phosphorylated AMPK (p-AMPKa) and total PPARa in the liver of mice. These results suggested that HTP may exert antihyperlipidemic activity through, at least in part, upregualtion of AMPK and PPARa.

\section{CONCLUSION}

The results presented in this study suggest that the flavone-rich B. chinensis leaf extract (HTP) has good potentials for lipid management. Up regulation of AMPK and PPARa are two possible mechanisms for its antihyperlipidemic activity.

\section{REFERENCES}

1. Raza J, Babb J, Movahed A. Optimal management of hyperlipidemia in primary prevention of cardiovascular disease. Int J Cardiol 2004; 97: 355366.

2. Patel A, Barzi F, Jamrozik K, Lam TH, Ueshima $H$, Whitlock G, Woodward, M. Serum triglycerides as a risk factor for cardiovascular diseases in the AsiaPacific region. Circulation 2004; 110: 2678-2686.

3. Boullart A, Graaf J, Stalenhoe A. Serum triglycerides and risk of cardiovascular disease. Biochim Biophys Acta 2012; 1821: 867-875.

4. Sinzinger $H$, Wolfram $R$, Peskar BA. Muscular side effects of statins. J Cardiovasc Pharmacol 2002; 40: 163-171.

5. Rosenbaum D, Dallongeville J, Sabouret $P$, Bruckert E. Discontinuation of statin therapy due to muscular side effects: A survey in real life. Nutr Metab Cardiovas 2013; 23: 871-875.

6. Benkhalti F, Prost J, Paz E, Jimenez F, Modafar C, Boustani E. Effects of feeding virgin olive oil or their polyphenols on lipid of rat liver. Nutr Res 2002; 22: 1067-1075.

7. Kim B, Ku C, Pham T, Park Y, Martin D, Xie L, Taheri R, Lee J, Bolling B. Aronia melanocarpa (chokeberry) polyphenol-rich extract improves antioxidant function and reduces total plasma cholesterol in apolipoprotein E knockout mice. Nutr Res 2013; 33: 406-413.

8. Qian $Q$, Liu X, He W, An Y, Chen Q, Wu J, Deng Y, Guo $L$, Zhang $Y$, Wang $T$. TG accumulation inhibitory effects of Jinqi formula by AMPK signaling pathway. J Ethnopharmacol 2012; 143: 41-48.

9. Guo P, Lian ZQ, Sheng LH, Wu CM, Gao J, Li J, Wang $Y$, Guo YS, Zhu HB. The adenosine derivative 2', 3', 5'-tri-O-acetyl-N6-(3-hydroxylaniline) adenosine activates AMPK and regulates lipid metabolism in vitro and in vivo. Life Sci 2012; 90: 1-7.

10. Hye Yang $M$, Vasquez $Y$, Ali Z, Khan IA, Khan SI. Constituents from Terminalia species increase
PPARalpha and PPARgamma levels and stimulate glucose uptake without enhancing adipocyte differentiation. J Ethnopharmacol 2013; 149: 490498.

11. Chao CY, Yin MC, Huang CJ. Wild bitter gourd extract up-regulates mRNA expression of PPARalpha, PPARgamma and their target genes in C57BL/6J mice. J Ethnopharmacol 2011; 135: 156-161.

12. Mahamuni S, Khose R, Menaa F, Badole S. Therapeutic approaches to drug targets in hyperlipidemia. Bio Med 2012; 2: 137-146.

13. Yoon M. The role of PPARa in lipid metabolism and obesity: Focusing on the effects of estrogen on PPARa actions. Pharmacol Res 2009; 60: 151-159.

14. Zhang $Y Y$, Wang $Q$, Qi $L W$, Qin $X Y$, Qin $M J$. Characterization and determination of the major constituents in Belamcandae Rhizoma by HPLCDAD-ESI-MS(n). J Pharm Biomed Anal 2011; 56: 304-314.

15. Wozniak D, Janda B, Kapusta I, Oleszek W, Matkowski A. Antimutagenic and anti-oxidant activities of isoflavonoids fromBelamcanda chinensis (L.) DC. Nutr Res 2010; 696: 148-153.

16. Kim Y, Yamada M, Lim S, Lee S, Ryu N, Shin K, Ohuchi K. Inhibition by tectorigenin and tectoridin of prostaglandin E2 production and cyclooxygenase-2 induction in rat peritoneal macrophages. Biochim Biophys Acta 1999; 1438: 399-407.

17. Jung SH, Lee YS, Lim SS, Lee S, Shin KH, Kim YS. Antioxidant activities of isoflavones from the rhizomes of Belamcanda chinensis on carbon terrachlorideinduced hepatic injury in rats. Arch Pharm Res 2004; 27: 184- 188.

18. Wu C, Li Y, Chen $Y$, Lao $X$, Sheng L, Dai R, Meng W, Deng $Y$. Hypoglycemic effect of Belamcanda chinensis leaf extract in normal and STZ-induced diabetic rats and its potential active faction. Phytomedicine 2011; 18: 292-297.

19. Chen Y, Wu CM, Dai RJ, Li L, Yu YH, Li Y, Meng WW, Zhang L, Zhang $Y$, Deng Y. Combination of HPLC chromatogram and hypoglycemic effect identifies isoflavones as the principal active fraction of Belamcanda chinensis leaf extract in diabetes treatment. J Chromatogr B Analyt Technol Biomed Life Sci 2011; 879: 371-378.

20. World Health Organization (WHO). Principles of Laboratory Animal Care. Chronicle 1985; 39: 51-56

21. Zhang $X, W u C, W u H$, Sheng L, Su Y, Zhang $X$, Luan $H$, Sun G, Sun $X$, Tian $Y$, Ji Y, Guo $P, X u X$. Antihyperlipidemic effects and potential mechanisms of action of the caffeoylquinic acid-rich Pandanus tectorius fruit extract in hamsters fed a high fat-diet. PLoS One 2013; 8: e61922.

22. Wu C, Shen J, He P, Chen Y, Li L, Zhang L, Li Y, Fu Y, Dai $R$, Meng $W$, Deng $Y$. The apha-glucosidase inhibiting isoflavones isolated from Belamcanda chinensis leaf extract. Rec Nat Prod 2012; 6: 110120.

Trop J Pharm Res, October 2014; 13(10): 1657 
23. Sharma B, Balomajumder $C$, Roy $P$. Hypoglycemic and hypolipidemic effects of flavonoid rich extract from Eugenia jambolana seeds on streptozotocin induced diabetic rats. Food Chem Toxicol 2008; 46: 23762383.

24. Narender $T$, Khaliq $T$, Puri A, Chander $R$. Antidyslipidemic activity of furano-flavonoids isolated from Indigofera tinctoria. Bioorg Med Chem Lett 2006; 16: 3411-3414.

25. Lee KT, Sohn IC, Kim DH, Choi JW, Kwon SH, Park HJ. Hypoglycemic and hypolipidemic effects of tectorigenin and kaikasaponin III in the
streptozotocin-Induced diabetic rat and their antioxidant activity in vitro. Arch Pharm Res 2000; 23: 461-466.

26. Xiong Y, Yang Y, Yang J, Chai H, Li Y, Jia Z, Wang Z. Tectoridin, an isoflavone glycoside from the flower of Pueraria lobata, prevents acute ethanol-induced liver steatosis in mice. Toxicology 2010; 276: 64-72.

27. Guo $Q$, Wang $P R$, Milot $D P$, Ippolito MC, Hernandez $M$, Burton CA, Wright SD, Chao Y. Regulation of lipid metabolism and gene expression by fenofibrate in hamsters. Biochim Biophys Acta 2001; 1533: 220232. 